\title{
Narrativa
}

\section{Visioni del mondo attraverso il lavoro. Le vite potenziali (2018) di Francesco Targhetta}

\section{Tiziano Toracca}

\section{(2) OpenEdition \\ Journals}

Edizione digitale

URL: https://journals.openedition.org/narrativa/365

DOI: $10.4000 /$ narrativa.365

ISSN: 2804-1224

\section{Editore}

Presses universitaires de Paris Nanterre

\section{Edizione cartacea}

Data di pubblicazione: 1 décembre 2019

Paginazione: 127-141

ISBN: 978-2-84016-350-3

ISSN: $1166-3243$

Notizia bibliografica digitale

Tiziano Toracca, «Visioni del mondo attraverso il lavoro. Le vite potenziali (2018) di Francesco

Targhetta», Narrativa [Online], 41 | 2019, online dal 01 septembre 2021, consultato il 08 décembre

2021. URL: http://journals.openedition.org/narrativa/365 ; DOI: https://doi.org/10.4000/narrativa.365

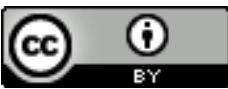

Narrativa est mise à disposition selon les termes de la Licence Creative Commons Attribution 4.0 International. 


\title{
Visioni del mondo attraverso il lavoro. Le vite potenziali (2018) di Francesco Targhetta
}

\begin{abstract}
RIASSUNTO
Il saggio considera Le vite potenziali (2018) di Francesco Targhetta alla luce di una serie di opere narrative pubblicate negli ultimi anni e lo analizza sulla base del doppio presupposto che affronti il tema del lavoro in maniera molto originale e che attraverso la rappresentazione del lavoro l'autore riveli aspetti emergenti e critici del presente. Il mondo del lavoro descritto nel romanzo è lo sfondo sul quale Targhetta proietta delle vicende private ma è anche l'esperienza che evidenzia e permette di cogliere la drammatica contraddizione tra le vite potenziali e le vite reali dei personaggi e più in generale tra il nostro immaginario e la realtà in cui viviamo.

\section{RÉSUMÉ}

Cet article se propose de réfléchir sur une série d'œuvres littéraires publiées ces dernières années en prenant en considération le roman Le vite potenziali (2018) de Francesco Targhetta, et en partant du double principe que ce texte affronte la thématique du travail d'une façon très originale, et qu'à travers la représentation du travail l'auteur révèle des aspects émergents voire critiques du présent. De fait, le monde du travail décrit dans le roman constitue un arrière plan où Targhetta projette des histoires personnelles, mais il représente aussi l'expérience qui met en évidence et permet d'identifier la contradiction dramatique entre les vies potentielles et les vies réelles des personnages, et, plus généralement, entre notre imaginaire et la réalité dans laquelle nous vivons.
\end{abstract}

\section{LA RAPPRESENTAZIONE DEL LAVORO}

Il mio intervento si riallaccia al doppio numero che Narrativa ha dedicato una decina d'anni fa al rapporto tra letteratura e azienda - numero cui va sicuramente riconosciuto il merito di aver riacceso l'interesse critico per il binomio letteratura-lavoro e di averlo aggiornato e problematizzato - e mira a inserirsi in 
un dibattito da qualche anno molto aperto e dinamico ${ }^{1}$. Non ricostruirò qui le tappe e i protagonisti di questo dibattito e neppure la storia, ormai trentennale, del rinnovato interesse che la letteratura italiana ha mostrato per il lavoro e per l'industria. L'ho fatto altrove e lo hanno fatto altri prima e dopo di mé2.

Vorrei qui richiamare brevemente tre aspetti metodologici che mi paiono importanti per affrontare questo tema e che sono più o meno condivisi da coloro che se ne occupano. In primo luogo, credo che il problema di come l'immaginario letterario contemporaneo abbia rappresentato il lavoro, la fabbrica e il paesaggio post-industriale tra la fine del xx e il primo ventennio del xxi secolo vada ricondotto all'interno del più ampio dibattito sulla tendenza al recupero di una funzione testamentaria, documentaria e di denuncia della letteratura, sulla presunta fine del postmoderno e il ritorno del reale, della realtà e del realismo. L'interesse per il lavoro e più in generale per le forme di produzione e di consumo e per le condizioni materiali dell'esistenza sono stati spesso invocati dalla critica letteraria come la riprova di una crescente attenzione per la realtà e per forme di racconto realiste o ipermoderniste e sono stati altrettanto spesso rivendicati da alcuni autori come il segnale di un nuovo impegno in campo letterario ${ }^{3}$. In secondo luogo, sono convinto che nell'immaginario letterario il tema del lavoro sconti radici profonde e tentacolari e sia più esteso e denso di figure $\mathrm{e}$ significati di quanto non sembri a prima vista e che perciò il senso della sua rap-

1. Contarini Silvia (a cura di), Letteratura e azienda. Rappresentazioni letterarie dell'economia e del lavoro nell'Italia degli anni 2000, in Narrativa, n. 31-32, 2010.

2. Mi limito a citare alcuni interventi pubblicati negli ultimi due o tre anni: BAGHETTI Carlo (a cura di), Letteratura e lavoro in Italia. Analisi e prospettive, Nóto5, n. 4, 2017; TorACCA Tiziano, "Flessibilità e precarietà nella letteratura italiana contemporanea: «Personaggi precari» di Vanni Santoni”, in Di Nunzio Novella, Jurišić Srecko, RaGni Francesco (a cura di), «La parola mi tradiva». Letteratura e crisi, Perugia, Culture territori Linguaggi - CTL Università di Perugia, n. 10, 2017, pp. 53-66; CETERONI Alessandro, La letteratura aziendale. Lavoro, fabbriche, uffici e precariato dalla fine del Novecento ad oggi tra romanqi, racconti, inchieste e poesia, Milano, Prospero, 2018; Summa Romano, La Littérature italienne et le monde du travail aujourd'bui, Paris, L'Harmattan, 2018; Pegorari Daniele Maria, Scritture precarie. Editoria e lavoro nella grande crisi. 2003-2017, Bari, Stilo editrice, 2018; ID., Il lavoro della letteratura. Forme, temi, metafore di un conflitto occultato e di un'emancipazione a venire, in L'Ospite ingrato, 2018; Condello Angela, Toracca Tiziano, I work, therefore I am. Law, Labour and the Humanities. Contemporary European Perspectives, London, Routledge, 2019 (in corso di stampa).

3. Oltre all'inchiesta di Allegoria n. 57 del 2008 curata da Raffaele Donnarumma e Gilda Policastro (Ritorno alla realtà? Otto interviste a narratori italiani) e all'ampio dibattito che ha fatto seguito, rimando alle riflessioni di Dominique Viart, Patrick Cingolani e Raffaele Donnarumma. Cfr. inoltre: Contarini Silvia, De Paulis Maria-Pia, Tosatti Ada (a cura di), Nuovi realismi: il caso italiano. Definizioni, questioni, prospettive, Massa, Transeuropa, 2016 (si vedano in particolare i saggi di Maurizio Ferraris, Donata Meneghelli e Davide Luglio). 
presentazione vada storicizzato in una prospettiva di più lunga durata che non risalga soltanto alla fine degli anni Ottanta e l'inizio degli anni Novanta (quando prende avvio, appunto, la cosiddetta letteratura postindustriale) o al binomio Balestrini-Levi o agli anni Cinquanta e Sessanta (al dibattito su letteratura e industria avviato da Vittorini, agli intellettuali olivettiani etc.), ma anche più indietro, alla discontinuità stabilita alla fine del XIX secolo dalla rivoluzione industriale con la quale, per molti aspetti, prende avvio anche in Italia la modernità ${ }^{4}$. L'immaginario letterario, ma forse a ben guardare l'immaginario collettivo tout court, tiene fermi moltissimi valori, problemi, parole d'ordine e vere e proprie visioni del mondo che prendono avvio durante o subito dopo quella rivoluzione. La possibilità di pensare il lavoro così come più o meno lo intendiamo oggi comincia da $1 i^{5}$. Il diritto del lavoro, ad esempio, prende avvio da quella frattura, e lo stesso discorso vale per le idee di alienazione, sciopero, rivoluzione o coscienza di classe così come le conosciamo e le adoperiamo ancora oggi. Alla svolta impressa dalla modernità si deve poi uno dei nessi simbolici destinati a caratterizzare più a lungo, con più intensità e con maggiore ambivalenza la nostra concezione $\mathrm{e}$ immaginazione del lavoro. Mi riferisco al nesso tra il lavoro che gli individui svolgono e la loro identità sociale; alla possibilità che gli individui hanno di far parte di una società e di orientarla, di emanciparsi e di riscattarsi dalle proprie condizioni sociali attraverso il lavoro, in qualità di lavoratori. Per quanto rozza e falsa, l'equivalenza tra ciò che siamo e ciò che facciamo si manifesta nel linguaggio quotidiano, nei documenti che produciamo e scambiamo, nelle relazioni sociali e nell'ordine morale. A partire dall'età moderna, la sfera pubblica della nostra esistenza è concettualizzata e visibile attraverso un apparato simbolico incentrato sul lavoro. In terzo luogo, penso che il dibattito su questo genere di rappresentazione non debba rinunciare né alla possibilità di interrogarsi sul rapporto tra i testi e l'extraletterario (tanto più che abbiamo a che fare molto spesso con scritture ibride, autobiografiche, testamentarie e che l'interesse per il lavoro è riemerso, storicamente, in concomitanza con conflitti sociali e trasformazioni storiche e parallelamente a interventi giuridici rilevanti) ${ }^{6}$ né ai vantaggi di approcci comparatisti e interdisciplinari (i primi, che io sappia, davvero rari) per

4. Cfr. almeno Musso Stefano, Storia del lavoro in Italia: dall'unità a oggi, Venezia, Marsilio, 2002, e BATTILossi Stefano, Le rivoluzioni industriali, Roma, Carocci, 2002.

5. Cfr. Gorz André, Metamorfosi del lavoro [1988], Torino, Bollati Boringhieri, 1992; Dagognet François et alii, Philosophie du travail, Paris, Les Belles Lettres, 2013; De Masi Domenico, Il lavoro nel XXI secolo, Torino, Einaudi, 2018.

6. Ne ho parlato qui: "Labour between Law and Literature: Historical Similarities and Critical Propositions on the Present”, in Pólemos, n. 11 (2), 2017, pp. 361-377. 
quanto i vari contesti e $\mathrm{i}$ vari discorsi vadano tenuti distinti - a ciascuno la sua logica - senza pretendere un'univocità che sarebbe, in fondo, ideologia.

In questo saggio mi occupo delle Vite potenziali di Francesco Targhetta sul presupposto che sia un'opera che tratta il tema del lavoro in maniera originale e che attraverso la rappresentazione del lavoro l'autore riveli aspetti emergenti e critici del nostro presente. Il romanzo di Targhetta va ad affiancarsi a una serie di opere sul lavoro davvero considerevoli (anche se molto diverse tra loro) che sono state pubblicate negli ultimi anni e che hanno smentito coloro che pensavano alla rappresentazione del lavoro (e in particolare del precariato) come a una moda passeggera (per quanto, va detto, l'interesse per il lavoro è stato anche questo). 108 metri. The new working class hero (2018) di Alberto Prunetti, già autore di Amianto (2012) e oggi direttore della collana Working Class di Alegre ${ }^{7}$; Ipotesi di una sconfitta (2017) di Giorgio Falco, autore del libro più sconvolgente e sperimentale sul lavoro precario, Pausa caffe (2004) ${ }^{8}$, e autore di Mondo macello (2009), uno dei racconti più raffinati sul tema del lavoro, dedicato in particolare all'invisibile e spietata pervasività del sistema di produzione e alla sua capacità di vegliare sul mondo dopo averlo reso docile e ubbidiente ${ }^{9}$; Works (2016) di

7. Cfr: https://www.wumingfoundation.com/giap/2018/11/collana-working-class/.

8. Ma non il più fortunato. L'opera sul lavoro precario che ha destato più attenzione è probabilmente Il mondo deve sapere. Romanzo tragicomico di una telefonista precaria (Einaudi, 2006) di Michela Murgia (anche per la trasposizione cinematografica che ne ha dato Virzi). Ma hanno segnato il campo anche Cordiali saluti (Einaudi, 2005) di Andrea Bajani e Mi chiamo Roberta, ho 40 anni, guadagno 250 euro al mese... (Einaudi, 2006) di Aldo Nove. Su Ipotesi di una sconfitta si trovano molte recensioni e commenti sul web, non molti articoli o saggi accademici. Cfr. Giglioli Daniele, "Soggetto, lavoro, precarietà. Ipotesi di una sconfitta di Giorgio Falco", recensione uscita sul Corriere della sera e ora disponibile su Le parole e le cose: http://www.leparoleelecose.it/?p=30835; la mia recensione apparsa su Allegoria, n. 78, 2018 (in corso di pubblicazione); il bel saggio di RAcCIS Giacomo, "«Il lavoro è ovunque»: forme del racconto e forme del potere nella narrativa di Giorgio Falco", in Ticontre, n. XII, 2019 (in corso di pubblicazione). Per una lettura di Ipotesi di una sconfitta in confronto a Works di Trevisan, cfr. SIRI Nicole, "Che cos'è il lavoro oggi. Works di Vitaliano Trevisan e Ipotesi di una sconfitta di Giorgio Falco", in Le parole e le cose, 4 maggio 2018, http:/ /www.leparoleelecose.it/?p=33580. Siri evidenzia la rilevanza dello scarto generazionale tra i due scrittori (Trevisan, 1960; Falco, 1967) e interpreta la loro diversa rappresentazione del lavoro come un riflesso di una cesura rapida e decisa avvenuta nel mondo del lavoro.

9. Falco Giorgio, Mondo macello, in Sono come tu mi vuoi. Storie di lavori, Roma-Bari, Laterza, 2009, pp. 141-148. "Il nostro consumatore deve solo pensare a vivere tranquillamente e svagarsi perché noi vegliamo sempre su di lui e gli prepariamo la bistecca, lo stipendio, il finanziamento agevolato per l'auto a metano conveniente, il corso d'inglese alla fine assolata di settembre, il volo economico di un penultimo momento, sette giorni su sette, ventiquattro ore al giorno, a Natale, a Ferragosto, sempre, anche in quelle sere nella quali la piccola amarezza quotidiana scompare brevemente per la 
Vitaliano Trevisan (a oggi probabilmente il capolavoro della narrativa sul lavoro, su questo ha ragione Gianluigi Simonetti) ${ }^{10}$; Addio: Il romanzo della fine del lavoro (2016) di Angelo Ferracuti, uno degli autori che con più costanza e passione ha riflettuto sul lavoro e lo ha rappresentato, a partire dalla raccolta di racconti Norvegia (1993) ${ }^{11}$; Cartongesso (2014) di Francesco Maino, un romanzo bello e potentissimo, che non è stato abbastanza letto e discusso ${ }^{12}$ : insieme alle Vite potenziali e in rapporto al tema del lavoro queste opere contribuiscono a creare un panorama di assoluto rilievo nella narrativa italiana contemporanea ${ }^{13}$.

voglia di festeggiare qualcosa, un campionato, un figlio, un ballottaggio" (p. 147). Le antologie di racconti sul lavoro pubblicate negli ultimi anni sono davvero molte e testimoniano un interesse autentico e diffuso per il lavoro. Non si può ignorare tuttavia che i testi di qualità, originali e non schiacciati su contenuti ideologici o morali, siano pochi.

10. In La letteratura circostante. Narrativa e poesia nell'Italia contemporanea (Il Mulino, 2018), Simonetti ha parlato di Works come del capolavoro giunto alla fine del ciclo di opere sul lavoro ma non è detto che il ciclo sia finito (e il romanzo di Targhetta rappresenta già una smentita). Sono d'accordo (Works è un capolavoro) perché in nessun'altra opera il lavoro è rappresentato in maniera così problematica e ambivalente: viene infatti rifiutato da un personaggio che però lavora moltissimo e bene (su questo ha ragione Mara Santi), non da un personaggio pigro, indolente, perdigiorno, ma da un personaggio iperattivo e intraprendente, capace di svolgere bene il proprio mestiere, pronto a rimboccarsi le maniche e a sacrificarsi. L'ambivalenza di Works deriva dal fatto che il lavoro viene sì rifiutato ma nella misura in cui è contrapposto all'operosità, all'espressione di sé e alla ricerca della verità. Sebbene sorretta da quella sorta di orizzonte trascendente che è la carriera di artista, la straordinaria euforia della voce narrante trasfigura la necessità del lavoro (da cui non si scappa) facendo sì che ogni esperienza, anche la più negativa, prenda valore. Su Works sono state date interpretazioni molto diverse a riprova della sua ambivalenza: cfr. TORACCA Tiziano, SANTI Mara, "La Procedura di Mobilità e la sua rappresentazione letteraria: Mobilità e Mobilità n. 2 in Works (2016) di Vitaliano Trevisan", in Forum Italicum (in corso di stampa); ConTARINI Silvia, "Refusal of Work in the Italian Literature. From Vogliamo tutto (Balestrini 1971) to Works (Trevisan 2016)", e Baghetтi Carlo, "Works by Vitaliano Trevisan and the Representation of Work in the Neo-Liberal Age", entrambi i saggi in I work, therefore I am. Law, Labour and the Humanities, cit. Cfr. anche l'intervista di Policastro a Trevisan, in Le parole e le cose, 15 aprile 2019, http:/ / www.leparoleelecose.it/?p=35363

11. Ne ho parlato estesamente qui: "«Regno della libertà» o «regno della necessità»? La narrativa italiana contemporanea di fronte all'ambiguità del lavoro. A partire da: Addio. Il romanzo della fine del lavoro (2016) di Angelo Ferracuti", in Il lavoro della letteratura. Forme, temi, metafore di un conflitto occultato e di un'emancipazione a venire, cit., pp. 177-193.

12. Cfr. la recensione di Moca Matteo, "Francesco Maino, Cartongesso", in Allegoria, n. 69-70, 2014, p. 419. Cfr. anche De Mieri Michele, "Il Nordest di Cartongesso. Un esordio importante: un punto di arrivo", http://www.minimaetmoralia.it/wp/cartongesso-francesco-maino/, 23 maggio 2014; IlletTERATI Luca, "Il farnetico, il dolore, e la vergogna. Una certa idea di Veneto", http://www.leparoleelecose.it/?p=14888, 26 dicembre 2015.

13. Il premio Biella "letteratura e industria" e il premio Volponi sono manifestazioni molto utili per approfondire lo "stato dell'arte" del binomio letteratura e lavoro. 
Francesco TARghetTA (1980): Le vite POTENZIALi (2018)

Diversamente dalle opere sopracitate di Maino, Trevisan, Ferracuti, Falco e Prunetti o da quelle che altri autori italiani hanno dedicato al lavoro e alla fabbrica (ad esempio Simona Baldanzi, Francesco Dezio, Giulia Fazzi, Eugenio Raspi, Emanuele Tonon o Stefano Valenti) il romanzo di Targhetta non è un'opera in tutto o in parte autobiografica ${ }^{14}$. L'autore, un narratore onnisciente, non rientra nel sistema dei personaggi e non c'è ragione per proiettare la sua ombra sulle vicende rappresentate ${ }^{15}$. Anche se l'ambientazione è ben definita (si parla di un'azienda informatica impiantata a Marghera) e nonostante l'autore (nato a Treviso) dimostri di conoscere a fondo le zone e il territorio che descrive e ammetta di essersi ispirato a situazioni e persone reali (come si legge nei ringraziamenti finali Targhetta è stato ospite di un'azienda, Alpenite, per qualche tempo) i personaggi e l'intreccio sono frutto d'invenzione.

Il mondo del lavoro che viene descritto ha poi una serie di caratteristiche piuttosto insolite rispetto a quanto è stato rappresentato dagli scrittori italiani negli ultimi decenni. Anzitutto, dei tre personaggi principali del romanzo (Luciano Foresti, Giorgio De Lazzeri, detto GDL, e Alberto Casagrande) i primi due sono assunti con contratti a tempo indeterminato e non sono perciò dei disoccupati o dei lavoratori intermittenti e precari (per quanto siano

14. Sulla matrice autobiografica come costante della narrativa sul lavoro rimando soprattutto a: ConTARini Silvia, "Raccontare l'azienda, il precariato, l'economia globalizzata. Modi, temi, figure", in Letteratura e azienda, cit., pp. 7-24 e PANELLA Claudio, "Raccontare il lavoro. Fiction, reportage e altre formule ibride a confronto nella letteratura italiana dell'ultimo decennio", in Somigli Luca (a cura di), Negli archivi e per le strade. Il ritorno alla realtà nella narrativa italiana di inižio millennio, Roma, Aracne, 2013, pp. 409-434.

15. Si tratta di un narratore onnisciente che articola i pensieri di tutti i personaggi e che interviene spesso con proprie riflessioni e con propri giudizi (per commentare, descrivere e puntualizzare). La somiglianza di fondo che caratterizza l'andamento al quale è sottoposta la mimesi dell'interiorità dei personaggi rappresentati (nonostante la loro grande diversità traspare sempre la sensibilità, unica, dell'autore) e l'insistenza riflessiva o dettagliante anche là dove il racconto potrebbe procedere più speditamente, in stile semplice, mi sembrano due limiti di un romanzo che è comunque molto ben congegnato e che mostra un investimento stilistico notevole. Rimando a due recensioni ragionate: Donati Riccardo, "L'ombra di Leopardi nell'era digitale. Le vite potenziali di Francesco Targhetta", Le parole e le cose, 18 aprile 2018, http://www.leparoleelecose.it/?p=33240, e Marsilio Morena, "I dagherrotipi contemporanei di Francesco Targhetta: dalle vite in posa di Perciò veniamo bene nelle fotografie alle esistenze frenetiche di Le vite potenziali", La letteratura e noi, 18 luglio 2018, https://www.laletteraturaenoi.it/index.php/ interpretazione-e-noi/820-i-dagherrotipi-contemporanei-di-francescotarghetta-dallevite-in-posa-di-perciò-veniamo-bene-nelle-fotografie-alle-esistenze-frenetiche-di-levite-potenziali.html 
comunque dei lavoratori flessibili), il terzo è il fondatore e il direttore dell'azienda che da lui prende il nome (la Albecom) ed è perciò un imprenditore in carriera $^{16}$. Tutti piuttosto giovani e benestanti, inoltre, i personaggi sono legati tra loro non solo da rapporti professionali ma anche da rapporti personali e confidenziali (esemplare il rapporto tra Alberto e Luciano ma anche il "meeting al Molo Cinque di Marghera"17), svolgono dei lavori a basso rischio ${ }^{18}$ (programmatore informatico, pre-sales e direttore d'azienda, mestieri che per quanto estremamente diffusi in epoca digitale restano tra l'altro ancora poco rappresentati in campo letterario) e adoperano un linguaggio standard infarcito però di anglismi e acronimi che ne fanno una sorta di slang che rimanda ora alla cultura nerd e geek (Luciano e Alberto), ora al culto manageriale per l'efficienza, la leadership e l'“ottimizzazione" della vita (Alberto), ora ai riti adrenalinici, tossicomani e priapeschi che caratterizzano l'alea dei grandi affari (GDL), un mondo quest'ultimo in cui è necessario persuadere gli altri dominandoli, imporsi un entusiasmo e un'euforia simulati, sapersi fabbricare una controfigura virtuale disposta ad abbandonarsi a ogni evenienza ${ }^{19}$. L'azienda al centro della rappresentazione, infine, è proficua e in ascesa (fattura seicentomila euro al mese e continua ad assumere personale), per quanto la concorrenza e il mercato siano spietati e nonostante abbia sede in una landa squallida e desolata.

16. "La Albecom era stata fondata ed era presieduta da Alberto Casagrande, trentaquattro anni, capelli castani, statura media, qualche riga incipiente attorno agli occhi, apprezzata abilità nell'assemblare mobili Ikea, una particolare passione per la buona tavola [...] e il culto della chiarezza"; TARGHETTA Francesco, Le vite potenziali, Milano, Mondadori, 2018, pp. 8-9.

17. Ibid., p. 95 e ss.

18. All'inizio del romanzo, di fronte all'avviso appeso alla porta di una chiesa ("la 'Chiesetta dell'Agip"') nel quale si legge che le mura interne dell'edificio sono ricoperte "dall'elenco dei morti sul lavoro al Petrolchimico", Luciano pensa: "Chissà se i programmatori possono morire sul lavoro". È il primo pensiero in forma diretta che troviamo nel testo. "Accostato al loro [a quello svolto dai lavoratori del Petrolchimico], il suo mestiere sembrava un passatempo" (Ibid., pp. 7-8). È in questo stesso frangente che veniamo a sapere che il personaggio ha un contratto di lavoro a tempo indeterminato (Ibid., p. 8).

19. Dell'interesse per la figura del nerd e del suo legame con l'inetto primonovecentesco (e di molte altre cose) ha parlato lo stesso Targhetta in varie interviste tra cui ad esempio quella curata da Nicola De Cilia: https://www.premiocomisso.it/le-vite-potenziali-nicola-de-cilia-intervista-francesco-targhetta/, 26 agosto 2018. La più bella definizione del nerd l'ha data, secondo Targhetta, Carlo Levi nell'Orologio descrivendo la figura di Martino. Di tossicomania in rapporto al personaggio di GDL ha parlato CoRLito Beppe, "Due romanzi sul tema del lavoro", in La letteratura e noi, 20 novembre 2018, https://www.laletteraturaenoi.it/index.php/interpretazione-e-noi/864-due-romanzi-sul-tema-del-lavoro.html 
È proprio pensando ai non-luoghi e agli spazi in cui i personaggi lavorano e si incontrano, ai tragitti che fanno, al territorio desertificato in cui si muovono e in cui abitano che la continua promessa di vite potenziali in cui consiste la loro attività, l'e-commerce, suona sinistra e drammaticamente illusoria.

Solo nei luoghi desolati certe vite possono trovare la loro armonia: i bar decadenti, le panchine lungo la circonvallazione, le piazze di periferia con le fontane disseccate e il cemento dei palazzi a cintura, le strade sporche dietro la stazione. Marghera. ${ }^{20}$

La descrizione dell'area in cui si trova l'appartamento di Alberto e Paola e la descrizione del territorio in cui è in funzione il termovalorizzatore di Fusina ${ }^{21}$, a sud di Marghera (le spianate deserte gonfie di morbi e veleni, le fabbriche dismesse, le pile di container, i cantieri aperti, le campagne da bonificare, il trans appostato alla rotonda dell'Enel), esprimono molto bene l'abbrutimento assoluto del paesaggio in cui i personaggi vivono e si muovono ${ }^{22}$.

Basate non a caso "sulla delocalizzazione e sulla desincronizzazione", le "ipotesi e opzioni di consumo", le "possibilità di esperienza" e "di una vita più ricca [...] pronta a diventare più intensa, sempre sul punto di esplodere, di farsi più vasta e desiderabile" ${ }^{23}$ riguardano in fondo, e anzitutto, proprio i personaggi. Sono le vite di Alberto, Luciano e GDL a subire il ricatto della potenzialità, della simulazione e dell'illusione che essi vendono e a rimanere però congelate, rinchiuse dentro un carrello virtuale, sul punto di esplodere altrove e di compiersi in un altro tempo. Sono anzitutto le loro vite a essere rinviate e a restare nel frattempo confinate allinterno di un mondo piccolo e desolante ${ }^{24}$.

20. TArghetta Francesco, Le vite potenziali, cit., p. 93.

21. Ibid., rispettivamente p. 25 e p. 124.

22. Nel finale, il paesaggio che si vede dal nuovo ufficio della Albecom "ricorda un po' Černobyl" (Ibid., p. 241). Raoul Bruni ha parlato di "scenario quasi post-apocalittico" e ha ricordato come Targhetta avesse già scritto "un suggestivo reportage poetico" su queste zone apparso sulla Lettura del Corriere della Sera. Cfr. BRuni Raoul, "Percorsi esistenziali sullo sfondo di Marghera: Francesco Targhetta", minima\& moralia, 20 aprile 2018, http://www.minimaetmoralia.it/wp/marghera-francesco-targhetta/

23. Sono tutte espressioni ricavate dal discorso che Alberto rivolge all'architetto Godin mentre gli spiega che cosa fa Albecom e qual è la sua mission. É senza dubbio una tra le pagine migliori (e più importanti) del romanzo (TARGHETTA Francesco, Le vite potenziali, cit., pp. 54-55).

24. Come i colleghi del Vega, i quali “avevano l'impressione di un incarceramento quotidiano mascherato da privilegio" (Ibid., p. 13). 
Uno degli aspetti più straordinari del romanzo di Targhetta è proprio il continuo straniamento provocato dall'accostamento del paesaggio postindustriale (le rotaie inutilizzate di Via delle Industrie, gli alberi mutili, le insegne arrugginite di attività cessate, il cemento) agli altisonanti "campi d'azione dell'azienda" (e-commerce, mobile, portals, strategy)" 25 . I grandi obiettivi di Albecom vengono programmati e raggiunti lavorando in un ambiente deantropizzato: pieno di immondizia, di relitti architettonici, di scarti prodotti da un sistema di produzione e da forme di consumo rapinose e distruttive che se affondano le loro radici in un passato industriale in decadenza di cui il Petrolchimico di Porto Marghera è l'emblema più lampante, riaffiorano tuttavia continuamente nel presente. Un paesaggio dunque deturpato, reso ancora più squallido da una quotidianità incurabile fatta di gesti automatici, azioni impersonali e inespressive, oggetti di cattivo gusto (il carillon che suona Per Elisa) e scadenti (l'abat-jour finto Tiffany).

Il mondo del lavoro rappresentato da Targhetta fa anzitutto da sfondo a una vicenda privata, quella specie di triangolo amoroso di cui sono protagonisti Alberto, Luciano e GDL. Tutti loro in qualche modo si desiderano e si inseguono vicendevolmente. Luciano, che per le sue capacità professionali e la sua docilità è apprezzato sia da Alberto (Albecom) sia da GDL (Altobello) ma che nello stesso tempo, per la sua cronica inettitudine e la sua asocialità patologica è da entrambi tenuto a distanza, insegue a sua volta Alberto (perché vorrebbe essere stimato dall'amico in qualità di amico, alla pari) e GDL (perché in fondo, qualche volta, vorrebbe poter essere irresponsabile, cinico e potente come lui). GDL vorrebbe essere come Alberto (almeno professionalmente: un imprenditore e non un subordinato) e Alberto vorrebbe che GDL gli fosse fedele e non lo tradisse come invece poi accade. Il nocciolo del romanzo è proprio questo: l'improvviso tradimento di GDL ${ }^{26}$, la sua scoperta $(\text { casuale })^{27}$ da parte di Alberto e l'obbligo di scegliere da che parte stare rimesso

\section{Ibid., p. 14.}

26. Il tradimento di GDL comincia a profilarsi quasi subito, dopo una sessantina di pagine.

27. È l'architetto Godin, chiamato a fare progetti per entrambe le società, a far scoprire ad Alberto il tradimento di GDL. A questo personaggio Targhetta affida un interessante commento dissacratorio sulla fine del lavoro e sull'introduzione del reddito di base. "Godin confessò che aveva letto da poco un articolo sulla prospettiva della fine del lavoro [...] Reddito base per tutti e tempo libero a profusione: cos'era, la parodia hippie dell'antropocene? Ormai per giustificare il cazzeggio si tiravano in ballo la tecnologia, le stampanti 3D, i robot che provano emozioni, i sistemi open source, il finto fai-da-te ovunque, e tutta un'interpretazione fricchettona di internet palesemente 
infine a Luciano. All'insaputa di Alberto, GDL decide ${ }^{28}$ di lasciare la Albecom e di fondare insieme a Mariotto (il sobillatore) e Barbero (il finanziatore) una propria azienda (l'azienda Altobello) che offra servizi analoghi nel campo dell'e-commerce. Decide cioè di farsi imprenditore come Casagrande e di sottrarre alla Albecom non soltanto alcuni affari già avviati (Vileda ad esempio ${ }^{29}$ ) ma anche i suoi migliori lavoratori. Tra questi è Luciano, uno dei più capaci programmatori informatici in forza da sempre alla Albecom. Un tradimento vero e proprio dunque (e in questi termini lo definisce Alberto) considerando il modo subdolo e meschino con cui GDL immagina di sfruttare fino in fondo l'impresa in cui lavora, derubandola di guadagni e di personale, per poi abbandonarla (GDL progetta di continuare a lavorare in Albecom per un po' di tempo come socio occulto); considerando che la "fuga" del personale formato e competente era stata la prima grande preoccupazione manifestata da Alberto ai suoi collaboratori (è su questo monito che si apre il romanzo e in particolare la rappresentazione dell'azienda ${ }^{30}$; considerando infine che il rapporto tra Alberto e Luciano, per quanto si sia ormai rarefatto e sfilacciato (come sanno entrambi e come intuisce anche GDL) ${ }^{31}$, rimonta agli anni del liceo e affonda le sue radici in alcune esperienze mitiche che riaffiorano spesso alla mente dei due ${ }^{32}$.

autoassolutoria. 'Sempre pronta, certa gente, a tirare fuori la democrazia per non fare una sega"” (Ibid., p. 106).

28. A modo suo e cioè istintivamente: "GDL sembrava aver già preso la sua decisione, in modo del tutto istintivo, senza aver minimamente approfondito l'argomento con Mariotto, valutato la reale consistenza del patrimonio, considerato i pro e i contro, discusso con Veronica, saputo chi fosse il terzo uomo" (Ibid., p. 70).

29. Cfr. Ibid., pp. 128-131.

30. Durante la prima vera scena aziendale del romanzo, la riunione operativa organizzata da Alberto, quest'ultimo si lamenta delle ripetute "fughe" del personale verso altre aziende. "Allora, sapete che anche Fulvio se n'è andato [...] Così non va. Assumiamo personale, lo formiamo, gli passiamo competenze e poi lo lasciamo andar via [...] La retention è un punto chiave. I nostri dovrebbero dire: 'Duecento euro in più? Chissenefrega! Preferisco rimanere qua"” (Ibid., pp. 9-10).

31. "Certo, è vero che Luciano e Alberto si conoscevano dai tempi del liceo, ma ormai sembrava non rimanere nulla della loro vecchia complicità (se mai c'era stata). Erano colleghi; ed essere colleghi è un'altra storia, tipica dell'età adulta, quando l'unica cosa che si può avere in comune con qualcun altro è ciò che nella vita non si è riusciti a fare" (Ibid., p. 192). GDL è qui interessato a capire se Luciano possa o meno aver spifferato il suo piano ad Alberto (a capire se sia stato, a sua volta, un traditore) ma dice qualcosa di vero sul rapporto tra i due.

32. Cfr. Ibid., pp. 17-18. 
Ma il mondo del lavoro rappresentato da Targhetta non è soltanto lo sfondo sul quale l'autore proietta delle vicende private. È anche l'esperienza che evidenzia e permette di cogliere la contraddizione sopra descritta tra le vite potenziali e le vite reali dei personaggi e più in generale tra il nostro immaginario e la realtà in cui viviamo. È perciò l'esperienza che insieme al destino dei personaggi rivela un destino che ci riguarda. Tra la vita reale e la vita potenziale che i personaggi vivono - è proprio questa seconda vita, potenziale, che il loro lavoro contribuisce a fabbricare e vendere - c'è una contraddizione fortissima che però è divenuta ai loro occhi così abituale e naturale da non essere più percepita come tale. Si è spenta. La sproporzione tra la realtà quotidiana (spazi, abitudini, linguaggio, relazioni) e la spettacolarizzazione impressa nel nostro immaginario da ipotesi di consumo e da possibilità di esperienza delocalizzate e desincronizzate è un fenomeno di cui facciamo esperienza ogni giorno e di cui il web è forse l'ipostatizzazione più evidente. Alla realtà, concreta e finita, si contrappone la virtualità, astratta e infinita. Ma come dicevo è una contraddizione spenta: uno stato di natura accettato. Così Alberto, nel tentativo di capire perché GDL lo abbia tradito:

È un mondo, quello online, in cui si continuano a fare le stesse cose che si fanno nell'altro, ma senza rendersi conto di essere visti, e quindi con più disinvoltura, senza freni inibitori, ma con più fame e crudeltà ma con meno fatica, al punto da sviluppare il desiderio di provare tutto. E tutto, d'altronde, si mostra raggiungibile [...] Quasi tutto, adesso, può essere conquistato da casa, o anche dal bar (perché possedere una casa?), sicché, nascondendosi negli altri, si può ottenere il risultato di nascondersi a se stessi [...] fino all'ipotesi estrema di una perdita di sé nel flusso autistico in cui si muterebbe il mondo là fuori, dove ogni essere umano, ridotto a slombato automa, delegherebbe la propria esistenza alla controfigura virtuale [...] Internet ha regalato a tutti l'impressione di vivere più vite assieme e di essere più di se stessi soltanto. Com'è possibile accettare la singolarità del vecchio mondo dopo che si è stati iniziati all'infinità delle evenienze offerte da quello nuovo? GDL lo aveva tradito, ma semplicemente perché aveva trapiantato la forma mentis virtuale nel mondo di sempre ${ }^{33}$.

Oltre ai luoghi in cui i personaggi vivono, ci sono almeno altri due aspetti, nel testo, che danno conto del peso della realtà e della sua rimozione. Il primo riguarda il linguaggio e la comunicazione. Nelle Vite potenziali assistiamo a un continuo non-dialogo e a una persistente, reciproca assenza. Le frasi che $i$ personaggi si rivolgono stancamente, a pezzi e bocconi, sono spente, brevi, 
sospese. Basterebbe pensare al dialogo tra GDL e Luciano, in macchina, dopo la nuotata del primo nella pozza del canale industriale nord prima della riunione aziendale; al dialogo tra Alberto e Luciano in auto mentre vanno a Salisburgo; a quello, decisivo, tra Alberto e GDL dopo che il primo ha scoperto il voltafaccia del secondo ${ }^{34}$. Ma lo stesso discorso vale per i dialoghi tra i personaggi principali e tutti gli altri: tra Alberto e Paola (la moglie), tra GDL e Veronica (la fidanzata), tra GDL e Bea (l'amante), tra Alberto o Luciano da un lato e Matilde dall'altra parte. Solo gli slang (di vario tipo, come dicevo) colorano il linguaggio ma si tratta pur sempre di increspature modulate e strumentali, di un copione da recitare, imparato per imitazione. Se tutti o quasi tutti i personaggi sono a modo loro soli, infantili (Forza quattro) e maniacali (tutti hanno dei tic ossessivi) è perché sono inespressivi e conformisti, abbrutiti e afasici. Il secondo aspetto riguarda il passato. A ben vedere nessuno ha un buon rapporto con il passato. I genitori di Luciano sono un peso e sembrano dei morti in vita (il ricordo di Olga, l'antica collega e amica della madre che a un certo punto muore, è impressionante proprio perché la sua immagine si sovrappone a quella della madre ancora viva). GDL ha un padre (da cui ha imparato a non scomporsi mai) ma è un uomo senza passato e più in generale senza legami. La casa che Alberto ha ereditato dai suoi genitori (prematuramente scomparsi) e che è ormai abbandonata, marcia, trascurata, piena di ortiche e "adatta, ormai, solo per rappresentazioni simboliche della decadenza" 35 è un peso fastidioso, una responsabilità troppo reale. La cancellazione del passato rappresenta l'ennesimo tentativo di rimuovere la realtà con tutta la sua impurità e la sua dose di concretezza, problematicità e finitudine. L'unica relazione che sembra sempre sul punto di riemergere dal passato, l'amicizia tra Alberto e Luciano - nata sognando "attorno a tavoli ricoperti di cavi e $\mathrm{pc}^{\prime \prime 36}$ - è soggetta a reticenze continue e verrà infine superata. Non c'è dubbio che Alberto consideri la mossa di GDL un tradimento anche perché ha paura di perdere Luciano (soffiare il migliore programmatore alla Albecom fa appunto parte del piano Altobello) e tuttavia nel lungo viaggio in auto verso

34. Cfr. Ibid. rispettivamente p. 34, pp. 194-198 e pp. 204-206.

35. Ibid., p. 51.

36. "conobbero la gloria della manodopera informatica, si ubriacarono per le prime volte attorno a tavoli ricoperti di cavi e pc, si passarono le prime canne pensando alle frasi da scrivere nei ringraziamenti, sognarono di essere notati e assunti da qualche multinazionale di software che avevano boicottato, e al motto Being too smart ain't a big deal incarnarono, peraltro in piena coscienza, la più autentica declinazione di Leopardi nell'era digitale" (Ibid., p. 18). 
Salisburgo, anziché aprirsi e confidarsi con l'amico, che pure aveva colto il suo strano nervosismo e che per questo aveva provato a riaprire la "porta del loro passato", Alberto lo deride e lo tratta male ("Niente. Alberto non riusciva, con lui, a non essere uno stronzo" ${ }^{37}$ ). Poco dopo, in hotel, pur sentendo di dovergli parlare ${ }^{38}$ non lo fa e di quel dialogo non c'è traccia ${ }^{39}$. Il passato è troppo effettivo e tangibile per essere affrontato.

Accanto alla storia triangolare e tutta maschile ${ }^{40}$ sopra descritta ne scorre un'altra, meno centrale ma forse più importante in rapporto alla conclusione del romanzo. È la storia di Matilde, la barista dell'Incrocio (il bar frequentato dai lavoratori della Albecom e della zona) che studia svogliatamente Lingue Orientali e che dopo essere stata lasciata da Fulvio e dopo aver scoperto di essere incinta di quest'ultimo, chiede aiuto a varie persone tra cui Veronica e Alberto, finendo per confidarsi con Luciano. Questa storia non è importante perché Matilde aspetta un figlio (un figlio lo aspetta anche Paola ed è probabile che lo avranno anche GDL e Veronica) o perché rappresenti un personaggio particolarmente originale, al contrario. È importante perché Matilde riesce a prendere coscienza della propria infelicità e a desiderare un altro futuro ${ }^{41}$ e perché, in qualche modo, la sua storia provoca e prefigura il cambiamento di Luciano ${ }^{42}$. Il finale del romanzo è aperto (come ha dichiarato anche l'autore) ma a me pare che segni una svolta. A un certo punto, sebbene fugacemente e per interposta persona, sia Matilde che Luciano pensano al suicidio. La prima dalla torre del termovalizzatore di Fusina in cui lavora Luca, un caro amico che fa il gruista

37. Ibid., p. 197.

38. "A Luciano doveva parlare: doveva convincerlo a rimanere in Albecom. Erano o non erano amici dal liceo? Voleva un aumento? Meno trasferte? L'auto aziendale? Luciano non lo poteva tradire" (Ibid., p. 217).

39. Come si intuisce, Alberto si apre con Luciano nel viaggio di ritorno e tuttavia lo $\mathrm{fa}$ in maniera indiretta e distaccata. La riprova viene dal successivo dialogo tra Alberto e Paola (Ibid., pp. 234-235). Il destino dei due amici è ormai diviso e il loro passato è rimosso.

40. La moglie di Alberto, Paola, ha un ruolo piuttosto marginale (anche nel finale, quando dice ad Alberto di aspettare un figlio, l'interesse narrativo è per la bega in cui si è trovata Albecom non per lei) come del resto la fidanzata "ufficiale" di GDL, Veronica. Un ruolo in parte diverso ha invece l'amante di GDL, Bea, perché fa parte dello stesso "ambiente" di lavoro (è la "commerciale di SAP-Italia") e dunque perché contribuisce a raffigurare il tipo di relazioni virtuali e illusorie che quell'ambiente produce e postula.

41. Cfr. Ibid., pp. 230-231.

42. Un po' banale forse, ma è significativo che Matilde scelga di chiamare suo figlio Luciano. "Non sono gli esclusi, alla fine, ad avere ragione?”, Ibid., p. 231. 
(il lavoro "più solitario" e "più spaventoso" che avesse mai visto ${ }^{43}$ ), da dove la ragazza vede davanti a sé un'immensa pattumiera; il secondo dalla rupe del Mönchsberg, dallo strapiombo che sembra "suggerire cosa fosse la morte, lei sì possente e razionale, perché mai suscettibile di ripensamenti" e cioè da un "typical suicide site" come gli spiega un fotografo tedesco incontrato lì per caso, un uomo ossessionato dal suicidio e originario di "un paese vicino al viadotto di Göltzsch, da dove ogni anno si buttavano decine di persone"44. Dopo aver dovuto (volente o nolente) pensare al suicidio, Matilde e Luciano prendono coscienza del loro stato di minorità e reagiscono: Matilde capisce di non amare Fulvio e si domanda come fare a crescere diversamente suo figlio; Luciano compra "un bell'appartamento in centro a Mestre", prende con sé una gatta (che non può non far pensare ai randagi che si aggirano all'inizio del romanzo intorno alla chiesetta dell'Agip), esce ogni tanto con Matilde (di cui, è chiaro, si sta innamorando), è felice e "le vite degli altri, col passare del tempo, gli sembrano sempre meno desiderabili”"

Quanto al lavoro può benissimo non importargli più. Le giornate indeterminate del suo autunno, così vuote agli occhi altrui, a lui sembrano contenere tutto. E se gli capita di pensare ad Alberto e GDL, Luciano se li figura in una calca distante dai suoi spazi di incolto, ed è quasi sicuro che non li risentirà per molto tempo, forse mai più ${ }^{46}$.

Il finale del romanzo come dicevo è aperto ma il periodo appena citato, insieme ad altri dettagli (la barba lunga, l'andare "in giro", l'idea di poter resistere stando ai margini, a quarant'anni), lascia intuire che Luciano, probabilmente, si è licenziato dalla Albecom. Certo, ha ancora "impegni fuori città" - e questo è il motivo per cui esce ogni mattina dal parcheggio del centro di Mestre lasciando il posto auto a chi viene a lavorare in centro ${ }^{47}$ - ma sono impegni imprecisati. "Tutto, così, per lui è ancora possibile": se è vero che "mentre si asciuga i capelli col phon prima di uscire un sabato sera di novembre", il personaggio pensa alla "straordinaria potenza dell'immaginazione" vero che la potenza dell'immaginazione è qui reale e autentica e non astratta e

43. Ibid., p. 125.

44. Ibid., p. 219 e p. 221.

45. Ibid., pp. 239-240.

46. Ibid., pp. 240-241.

47. Ibid., p. 242.

48. Ibid., p. 240. 
illusoria. Di fatto, Luciano ha scelto di vivere la propria vita e non quella degli altri o di altri sé potenziali e questa scelta gioca proprio contro il suo vecchio lavoro, contro le vite potenziali che prometteva.

Tiziano Toracca Università di Torino 
